\title{
Role of tight junction-associated MARVEL protein marvelD3 in migration and Epithelial-Mesenchymal Transition of hepatocellular carcinoma
}

\section{Yanmeng Li ( $\square$ 18810676329@163.com )}

Capital Medical University Affiliated Beijing Friendship Hospital https://orcid.org/0000-0003-31517099

\section{Teng Li}

Chinese Academy of Medical Sciences Cancer Institute and Hospital: Cancer Hospital Chinese Academy of Medical Sciences

\section{Donghu Zhou}

Capital Medical University Affiliated Beijing Friendship Hospital

Jia Wei

Capital Medical University Affiliated Beijing Friendship Hospital

\section{Zhenkun Li}

Capital Medical University Affiliated Beijing Friendship Hospital

\section{Xiaojin Li}

Capital Medical University Affiliated Beijing Friendship Hospital

\section{Siyu Jia}

Capital Medical University Affiliated Beijing Friendship Hospital

\section{Qin Ouyang}

Capital Medical University Affiliated Beijing Friendship Hospital

\section{Saiping Qi}

Capital Medical University Affiliated Beijing Friendship Hospital

\section{Zhibin Chen}

Capital Medical University Affiliated Beijing Friendship Hospital

\section{Bei Zhang}

Capital Medical University Affiliated Beijing Friendship Hospital

\section{Jing Yu}

Capital Medical University Affiliated Beijing Friendship Hospital

\section{Jidong Jia}

Capital Medical University Affiliated Beijing Friendship Hospital

\section{Anjian Xu}

Capital Medical University Affiliated Beijing Friendship Hospital Jian Huang 


\section{Primary research}

Keywords: Hepatocellular carcinoma, marvelD3, EMT, migration, NF-kB

Posted Date: March 5th, 2021

DOl: https://doi.org/10.21203/rs.3.rs-274294/v1

License: (c) (1) This work is licensed under a Creative Commons Attribution 4.0 International License. Read Full License

Version of Record: A version of this preprint was published at Cell Adhesion \& Migration on January 1st, 2021. See the published version at https://doi.org/10.1080/19336918.2021.1958441. 


\section{Abstract}

\section{Background}

Tight junction (TJ) imbalance is associated with hepatocellular carcinoma (HCC). MarvelD3, which contains a conserved MARVEL (MAL and related proteins for vesicle trafficking and membrane link) domain similarly to occludin and tricellulin, is a recently identified integral membrane protein that forms TJs. However, little is known about the possible roles of marvelD3 in epithelial-mesenchymal transition (EMT) and metastasis of HCC. We aimed to demonstrate the role of marvelD3 in inhibiting HCC EMT and migration and further explore the underlying molecular mechanisms.

\section{Methods}

MarvID3 expression was assessed in HCC and normal liver tissues. Changes in marvelD3 expression were analyzed during transforming growth factor $\beta 1$ (TGF- $\beta 1$ ) and snail/slug-induced EMT. MarvelD3 knockdown HCC cell lines were established using marvelD3-siRNA to analyze correlations between marvelD3 and EMT-related proteins. Tumor cell behaviors were analyzed in marvelD3 knockdown HCC cells. Associations between marvelD3 and genes in the nuclear factor (NF)-KB pathway were also analyzed.

\section{Results}

Loss of marvelD3 expression was significantly correlated with the occurrence and TNM stage of HCC. MarvelD3 was downregulated in HCC cells with TGF- $\beta$ and snail/slug-induced EMT. Expression of marvelD3 protein was significantly associated with E-cadherin and vimentin in HCC cell lines. Knockdown of marvelD3 promoted tumor cell migration concomitant with activation of the NF-KB signaling pathway and increased matrix metallopeptidase 9 expression.

\section{Conclusions}

Our study demonstrated that MarvelD3 inhibited EMT and migration of HCC cells via NF-KB signaling pathway. This suggests that marvelD3 is a novel potential biomarker for the treatment and prognosis of HCC.

\section{Introduction}

Tight junctions (TJs), which are an intercellular adhesion complex of epithelial and endothelial cells, are very important for the growth of metastatic cancer cells. TJs separate the internal space of multicellular organisms from external compartments and form a diffusion barrier, which allows regulated movement of ions and solutes through the paracellular pathway [1, 2]. TJs consist of tight junction-associated marvel proteins (TAMPs) (occludin, tricellulin, and marvelD3) and claudins. Occludin, marvelD3 and claudin proteins are the major integral components of bicellular TJs [2, 3]. Claudins regulate the interactions between occludin, tricellulin, and marvelD3, which inversely modulate claudin 
oligomerization [2]. Their cross-linking functions have been associated with the regulation of signal transduction mechanisms that lead to epithelial cell proliferation and differentiation [4]. Moreover, deregulation of TJ expression has been reported in cancers, which indicates that they may be significant for tumorigenesis. However, the pathological significance of these observations is unclear [5-9].

MarvelD3 was recently discovered as a TAMP member. It has two isoforms and shows a broad tissue distribution. Knockdown of marvelD3 affects the paracellular barrier properties of TJs [1]. MarvelD3 functions as a regulator of epithelial cell proliferation, migration, and survival in human colon and pancreatic cancer cells $[6,10]$. Aberrant expression of claudin-3 has been shown to be significantly associated with the development and metastasis of various human cancers [9, 11-14]. Furthermore, it has been reported that marvelD3 interacts with claudin-3 [2].

Owing to the intrahepatic and extrahepatic metastases, hepatocellular carcinoma ( $\mathrm{HCC})$ is characterized by high recurrence and low 5-year survival rates [15]. It has been demonstrated that epithelialmesenchymal transition (EMT) plays a pivotal role in the early events of HCC [16]. There is accumulating evidence of a major link between EMT and tumor cell metastasis for strong motility and invasiveness [17]. Among all known signaling pathways involved in EMT, the transforming growth factor- $\beta 1$ (TGF- $\beta 1$ ) and snail-induced signaling pathways have been recognized to be responsible for the initiation, progression, and metastasis of HCC [18-21]. Claudin-3 inhibits EMT and invasion of lung squamous cell carcinoma cell [22]. Downregulation of marvelD3 is involved in EMT of human pancreatic cancer cells [6]. Additionally, some pathways, such as nuclear factor kappa B (NF-kB) and mitogen-activated protein kinase 1 pathways, have been investigated in EMT and metastasis of HCC, which perform biological functions through the interactions with a variety of cellular factors, including TGF- $\beta$ [23-25].

Previous studies have demonstrated that depletion of marvelD3 stimulates cell migration and proliferation of Caco-2 cells [10]. However, the novel role of marvelD3 in HCC remain unclear. In this study, we aimed to clarify the regulatory mechanisms of marvelD3 in HCC.

\section{Materials And Methods}

\section{Cell culture and human liver sample}

Human liver cancer cell line Hep3B was cultured in RPMI 1640 (Sigma, USA) supplemented with 10\% fetal bovine serum (FBS, Sigma), $100 \mathrm{U} / \mathrm{mL}$ penicillin, and $100 \mathrm{U} / \mathrm{mL}$ streptomycin at $37^{\circ} \mathrm{C}$ with $5 \% \mathrm{CO}_{2}$. Huh-7 cells were cultured in Dulbecco's modification of Eagle's medium (Sigma) with $10 \%$ FBS. Cells in the exponential growth phase were used in experiments.

All experiments involving human tissues were evaluated by the local ethics committee (Beijing You-an Hospital, Capital Medical University, Beijing, China, EC-B-031-A02-9.0). The sample was collected from a subject who provided informed consent for their tissue to be used for research purposes. For immunochemistry, normal liver tissue $(n=13)$ and liver tumor tissue $(n=25)$ were obtained as paraffin blocks and sectioned at $4 \mu \mathrm{m}$ thicknesses. Normal and tumor tissues were evaluated by two pathologists. 
Tissues for Western blotting $(n=4)$ and RT-PCR $(n=3)$, which included liver cancer $(C)$ and paired normal liver tissues $(\mathrm{N})$, were frozen tissue samples. The patient characteristics are listed in Table S1.

\section{Antibodies and reagents}

An anti-marvelD3 antibody (ab118916), anti-Claudin 3 antibody (ab15102) and anti-GAPDH (ab6276) were purchased from Abcam (USA). An anti-E-cadherin antibody (\#3195) and anti-vimentin antibody (\#5741) were purchased from Cell Signaling Technology (MA, USA). An anti-NF-kB antibody kit (\#9936) and anti- $\beta$-actin (\#3700) antibody was purchased from Cell Signaling Technology. Horseradish peroxidase (HRP)-conjugated goat anti-mouse and goat anti-rabbit IgGs were purchased from Zhongshan Jinqiao (Beijing China). TGF- $\beta 1$ (PeproTech, USA) was dissolved in citric acid ( $\mathrm{pH} \mathrm{3.0)}$ at a concentration of $10 \mu \mathrm{g} / \mathrm{mL}$, stored at $-20^{\circ} \mathrm{C}$, and diluted in culture medium to the required concentration of $10 \mathrm{ng} / \mathrm{mL}$. NF-kB inhibitor BAY 11-7082 (HY-13453; MCE) were added to the culture medium at a final concentration of $20 \mu \mathrm{M}$ in accordance with the manufacturer's instructions.

\section{siRNA-mediated knockdown of marvelD3 expression}

siRNA knockdown was performed using siRNA ID stB0014718A (RiboBio, Guangzhou, China) to target the marvelD3-coding region and negative control siRNA (ID siN0000001-1-5). Cancer cells were transfected with 20 nM siRNA using LTX reagent (Invitrogen, USA) in accordance with the manufacturer's instructions. The culture medium was replaced after 12 hours and subsequent assays were performed after 24 or 48 hours.

\section{Plasmid transfection}

The entire coding sequences of human snail and slug were inserted into the mammalian expression plasmid pcDNA3.1. Cells were transfected with the constructed plasmid using transfection reagent LTX (Invitrogen, USA) in accordance with the manufacturer's instructions. The culture medium was changed at 6 hours after transfection and the cells were harvested at 24 or 48 hours after transfection for subsequent assays.

\section{Cell survival assay and Transwell migration assay}

The effect of marvelD3 on HCC cells viability was determined by MTS cell proliferation assay kit (Promega, Madison, WI, USA) in accordance with the manufacturer's instructions. SiRNA-marvelD3 and siRNA-NC HCC cells $\left(5 \times 10^{3}\right.$ cells/well) were seeded into a 96-well plate. The OD value was read after 24 and $48 \mathrm{~h}$. For the migration assay, we used modified Boyden chambers with filter inserts (8 $\mu \mathrm{m}$ pore size). Approximately $1 \times 10^{5}$ cells in $200 \mu \mathrm{L}$ of medium were placed in the upper chamber, and $1 \mathrm{ml}$ of complete medium was placed in the lower chamber. After 12-36 h in culture, cells were fixed in 4\% paraformaldehyde for 30 min and then stained with $0.05 \%$ crystal violet in deionized water for $2 \mathrm{~h}$. Cells on the upper side of the filters were removed with cotton-tipped swabs and the filters were washed with PBS. Cells on the underside of the filters were viewed and counted under a microscope. 


\section{Wound healing assay}

HCC cells were seeded into a six-well plate and cultured overnight. The next day, after reaching approximately $\geq 90 \%$ confluence, three linear wounds were created using a $200 \mu$ l pipette tip. Cells were washed with PBS and cultured for $24 \mathrm{~h}$. Photographs of the wounded area were obtained immediately after making the scratch ( $0 \mathrm{~h}$ time point) and at $24 \mathrm{~h}$ to monitor the wounding healing capacity of the cells. The experiment was repeated at least once.

\section{Real-time PCR (RT-PCR)}

Total RNA was extracted from HCC cells and liver tissues using TRI Reagent (Sigma) in accordance with the manufacturer's instructions. A total of $2 \mu \mathrm{g}$ RNA was used for cDNA synthesis with a Reverse Transcription Kit (Roche, Germany) in a $20 \mu$ reaction. Equal amounts of cDNA were subjected to PCR using the following conditions: initial denaturation at $95^{\circ} \mathrm{C}$ for $10 \mathrm{~min}$, followed by 40 cycles of $95^{\circ} \mathrm{C}$ for $15 \mathrm{~s}$ and $60^{\circ} \mathrm{C}$ for $1 \mathrm{~min}$, and terminal extension at $72{ }^{\circ} \mathrm{C}$ for $5 \mathrm{~min}$. Each sample was examined in triplicate. GAPDH was used as the internal control. Real-time PCR primers are listed in supporting Table S2. GraphPad Prism v7 (GraphPad Software, San Diego, CA, USA) was used to create histograms.

\section{Western blotting}

Proteins were extracted from HCC cells and fresh liver tissue by lysis in RIPA buffer with protease and phosphatase inhibitors (Roche, USA). Equal quantities of proteins were separated by $12 \%$ SDS-PAGE and transferred onto a PVDF membrane (GE, USA) using a Bio-Rad wet transfer unit. After blocking with $5 \%$ $(\mathrm{w} / \mathrm{v})$ non-fat dried milk in TBST [25 mM Tris, $\mathrm{pH} 7.5,150 \mathrm{mM} \mathrm{NaCl}$, and 0.1\% (v/v) Tween-20] for $1 \mathrm{~h}$ at room temperature, the membrane was incubated with a primary antibody overnight at $4{ }^{\circ} \mathrm{C}$, followed by HRP-conjugated goat anti-mouse or goat anti-rabbit IgGs (1:5000) for $1 \mathrm{~h}$ at room temperature. Immunocomplexes on the membrane were visualized with Immobilon Western Chemiluminescent HRP Substrate (Millipore USA) using Image Lab Software (BIO-RAD).

\section{Immunofluorescence (IF)}

Treated HCC cells were washed three times in PBS, fixed for 30 min in $4 \%$ paraformaldehyde in PBS, and permeabilized for 10 min with $0.3 \%$ Triton X-100 in PBS. Non-specific binding sites were blocked by 60 min of incubation in $5 \%$ bovine serum albumin in PBS. Then, the cells were incubated with primary antibody against anti-E-cadherin or anti-vimentin (1:200) at $4{ }^{\circ} \mathrm{C}$ overnight. After three 5-min washes with PBS, the cells were incubated with a mixture of anti-rabbit Alexa fluor 488-conjugated secondary antibodies (1:200) for $2 \mathrm{~h}$ at room temperature. Following three 5-min wash with PBS, the cells were mounted on a slide in mounting medium with DAPI (Molecular Probe, USA). Cells were examined and photographed under a confocal microscope (FV 300, Olympus).

\section{Immunohistochemistry (IHC)}


Liver tissues were fixed in 10\% neutral buffered formalin, embedded in paraffin, and stained for routine histology. Sections were dewaxed in xylene and dehydrated in alcohol. Antigen retrieval was performed by microwaving in citric saline for $15 \mathrm{~min}$. Thin sections were deparaffinized and treated with $0.3 \%$ hydrogen peroxide for $15 \mathrm{~min}$ to block endogenous peroxidase activity. The sections were blocked further in $3 \%$ bovine serum albumin, followed by incubation with a primary antibody against E-cadherin (1:400) or marvelD3 (1:500) for $20 \mathrm{~h}$ at $4{ }^{\circ} \mathrm{C}$. After rinsing, the sections were incubated with a biotinylated secondary antibody for $60 \mathrm{~min}$ at room temperature. Protein expression was visualized by 3,3邓diaminobenzidine tetrahydrochloride staining. The sections were counterstained with hematoxylin before dehydration for $30 \mathrm{~s}$. Staining intensities were determined by measuring the integrated optical density (IOD) by light microscopy using Image-Pro v6.0.

\section{Statistical analysis}

All experiments were carried out at least three times. Data are represented as means \pm SEM. Differences between groups were calculated using the Student's t-test. Statistical significance was defined as $P<0.05$ for a two-tailed test. All statistical analyses were carried out with Graphpad Prism 7.0 software.

\section{Results}

\section{Associations of reduced marvelD3 expression with altered EMT markers in HCC tissues compared with normal liver tissues.}

In this study, we first confirmed differential MarvelD3 expression in HCC tissues vs. normal liver tissue. Western blot and RT-PCR assays revealed reduced marvelD3 expression in HCC tissue compared with paired adjacent normal tissues and a decrease of E-cadherin and increase of vimentin (Fig. 1A and B). IHC data were consistent with the western blot data. E-cadherin and marvelD3 protein levels were significantly lower in HCC tissues regardless of low or high grades compared with normal tissues (Fig. 1C and D), and lower expression of marvelD3 was observed in high TNM stage HCC tissue than in low grade (Fig. 1E).

\section{Downregulation of marvelD3 during TGF- $\beta 1$ and snail-induced EMT in HCC cells}

To detect the function of MarvelD3 in EMT of HCC cells, HCC cells (Hep3B and Huh-7) were stimulated with TGF- $\beta 1(10 \mathrm{ng} / \mathrm{mL})$ for $48 \mathrm{~h}$. Results showed that the TGF- $\beta 1$ treated cells exhibited remarkable morphological changes into spindle-shaped cells with loose cell connections (Fig. 2A) as well as reduced mRNA and protein expression of E-cadherin and marvelD3, while expression of vimentin was upregulated (Fig. 2B and C). During snail and slug-induced EMT of HCC cells, E-cadherin and marvelD3 proteins were decreased, while the vimentin protein level was increased (Fig. 2D).

\section{Effects of marvelD3 knockdown on regulation of EMT gene expression in vitro}

Many studies have revealed that EMT increases the incidence of cancer metastasis [26, 27]. Therefore, we ascertained whether marvelD3 reduced migration of HCC cells through regulation of EMT. We 
constructed marvelD3-knockdown HCC cells (Fig. 3A). Our results showed that the protein levels of Ecadherin were downregulated significantly, while those of vimentin were upregulated significantly in marvelD3 knockdown HCC cells (Fig. 3B). Consistent with the result of Western blot, IF showed reduced expression of Ecadherin and enhanced expression of vimentin in HCC cells after marvelD3 knockdown (Fig. 3C).

\section{Effects of marvelD3 knockdown on HCC cell migration in vitro}

EMT endows cells with migratory and invasive properties [28], thus we detected the effects of MarvelD3 expression on inhibition of HCC cell migration in vitro. The migratory effect of marvelD3 on HCC cells was assessed by wound healing and transwell migration assays. In marvelD3 knockdown HCC cells, tumor cell wound healing and their Transwell migration capacity were upregulated significantly (Fig. 4AD). However, marvelD3 knockdown did not affect tumor cell proliferation in vitro (Fig. 4E). These results demonstrated that marvelD3 reduced HCC cell migration.

\section{Effects of marvelD3 on inactivation of the NF-kB signaling pathway}

NF-KB, a master regulator of inflammation and cell death, may be a target for prevention or treatment of HCC [29]. Translocation of NF-KB-p65 into the nucleus and phosphorylation of IKK- $\beta$ and IKBa are markers of NF-KB pathway activation. Our western blot data revealed NF-KB pathway activation in marvelD3 knockdown HCC cells (Fig. 5A). We also analyzed the effect of marvelD3 on expression of NF$K B$ downstream genes such as MMP9 and found that marvelD3 knockdown upregulated the expression of MMP9 (Fig. 5A). We next used NF-kB inhibitor BAY 11-7082 to block activity of NF-kB in marvelD3 knockdown HCC cells (Fig. 5B). As a result, we observed reduced migration of marvelD3-silenced HCC cells treated with NF-KB inhibitor BAY 11-7082 (Fig. 5C). These data demonstrated that marvelD3 inhibited HCC cells migration partly through the NF-KB pathway.

\section{Discussion}

In this study, we found that marvelD3 was downregulated in HCC tissues and its downregulation was obviously correlated with the tumor stage. Additionally, our result suggested that TGF $\beta-1$ stimulation and snail or slug transfection reduced expression of marvelD3. Knockdown of marvelD3 changed EMT markers and induced migration of HCC cells. Mechanistic analysis suggested that marvelD3 inhibited HCC progression by inactivating the NF-KB pathway.

As a kind of tight junction (TJ)-associated protein, claudins are useful molecular markers for various cancers [30-32]. A recent study [33] has demonstrated that TJ protein expression is altered in HCC and cholangiocarcinoma. For example, CLDN1 has been shown to be upregulated in advanced liver disease and HCC, and differential CLDN4 expression facilitates distinguishing between these two forms of cancer at the molecular level. Another report also revealed that CLDN3 inhibits cancer aggressiveness and is a potential prognostic biomarker for HCC [9]. However, in the MARVEL family, occludin, tricellulin, and marvelD3, little is known about changes in their expression during cancer progression. MarvelD3 has 
been reported to play a significant inhibitor role in colon adenocarcinoma cells and pancreatic cancer cells $[6,10]$. In this study, we found that tumor tissue had lower expression level of marvelD3 than normal tissue and expression of marvelD3 was associated with TNM stage of HCC. Moreover, marvelD3 downregulation was found in human liver cancer cell lines Huh-7, HepG2, BEL7402, and Hep3B. These results indicate that marvelD3 plays a role as a tumor suppressor gene in tumorigenesis.

EMT is a multistep biological process whereby epithelial cells change by transient dedifferentiation to a mesenchymal phenotype. During carcinoma progression, EMT plays a crucial role in the early steps of metastasis when cells lose cell-cell contacts by ablation of E-cadherin. Strong inducers of EMT such as TGF- $\beta$ orchestrate both fibrogenesis and carcinogenesis. Additionally, the SNAI family with its members Snail (SNA1) and Slug (SNAI2) is involved in the activation of EMT. Snail is the prominent inducer of EMT in HCC [34]. In our study, we specifically examined changes in marvelD3 during TGF- $\beta$-induced EMT and overexpression of snail and slug in HCC cells. With downregulation of E-cadherin and upregulation of vimentin, expression of marvelD3 was decreased. A previous study has suggested that, under hypoxia and treatment with TGF- 31 , claudin-1 is decreased [6], and claudin-3 knockdown affects regulation of EMT gene expression in vitro [22]. Several other studies have shown that marvelD3 expression is more strongly reduced in cell lines with invasive phenotypes derived from breast, pancreatic, and prostate tumors than relatively normal cell lines[10]. Similar to previous studies, we established marvelD3 knockdown HCC cells by siRNA transfection and a significant change in EMT markers was observed in the marvelD3 knockout cell lines. These results indicate that marvelD3 plays an important role in the process of EMT and the further research of the correlation with marvelD3 and EMT may be valuable. The main characteristic of EMT is promotion of cell migration. Our data demonstrated that marvelD3 was indeed involved in EMT-related processes. Therefore, we speculated that marvelD3 inhibited migration of HCC cells. For verification, we compared the migratory capacities of marvelD3 knockdown and normal HCC cells. As a result, we observed a stronger migration capacity in marvelD3 knockdown HCC cells compared with the normal group. This result suggests that marvelD3 inhibits migration of HCC cells.

To further explore the mechanism of marvelD3 in the inhibition of EMT, we also carried out in-depth analysis of cell signaling pathway. The cross-talk between TGF- $\beta$ signaling and NF-KB is critical for various biological processes, including the development of different liver diseases $[24,35,36]$. The NF-KB signaling pathway stimulus to the regulation of hepatic fibrosis and HCC cell is recognized by receptors and then transmitted into the cell. This process is achieved through adaptor signaling proteins activating IKK. IKK phosphorylates IKB in the cytoplasm, which leads to degradation of IKB and release of NF-KB from the inhibitory complex. Subsequently, NF-KB protein translocates into nucleus where it binds to target sequences and activates gene transcription [37]. In our study, knockdown of marvelD3 increased phosphorylation of IKK- $\beta$ and IKBa and nuclear translocation of p65, which suggested that knockdown of marvelD3 activated the NF-KB pathway and further promoted expression of NF-KB downstream target gene MMP9. To verify this result, NF-KB activity inhibitor BAY 11-7082 was used to treat cells and the result demonstrated that inhibition of marvelD3 in HCC cell migration was partly mediated through the NF-KB pathway. However, it is a limitation that we had not further investigated other effective signal pathways, which could be involved in the effect of marvelD3 on HCC progress. 
In summary, our study showed that expression of marvelD3 was downregulated in human HCC tissues and HCC cells with TGF- $\beta 1$ /snail-induced EMT. In vitro experiments revealed that marvelD3 inhibited EMT and invasion of HCC cells via suppression of the NF-KB signaling pathway. Therefore, marvelD3 may be an effective inhibitor of EMT and migration in human HCC cells. This suggests that marvelD 3 can be further evaluated as a novel biomarker to predict the prognosis of $\mathrm{HCC}$ and as a therapeutic target for of HCC.

\section{Abbreviations}

HCC, Hepatocellular carcinoma; TJ, Tight junction; MARVEL, MAL and related proteins for vesicle trafficking and membrane link; EMT, Epithelial-mesenchymal transition; NF-KB, Nuclear factor kappa B;

TAMPs, Tight junction-associated marvel proteins; TGF- $\beta 1$, Transforming growth factor- $\beta 1$; MMP9, matrix metallopeptidase 9; RT-PCR, Real-time PCR; IHC, Immunohistochemistry; IF, Immunofluorescence.

\section{Declarations}

\section{Acknowledgements}

We thank Mitchell Arico from Liwen Bianji, Edanz Group China (www.liwenbianji.cn/ac), for editing the English text of a draft of this manuscript. The authors would like to thank Dr Yanjun Shen (Beijing Ditan Hospital Capital Medical University) for kindly providing the pathological section, and Dr Guangyong Chen (Department of Pathology, Beijing Friendship Hospital, Capital Medical University) for critical pathological review.

\section{Authors' contributions}

$\mathrm{JH}, \mathrm{AJ} \mathrm{X}$ and JD J conceptualized and supervised the study; YM L and TL performed the most experiments; ZK L, DH Z and XJ Lprovided technique supports. JW acquired and managed patients. SY J and Q OY were responsible for RT-PCR. SP Q, ZB C and BZ participated in Western blotting. JY provided clinical analysis and professional assessment. $Y M L$ and $T L$ wrote the manuscript. All authors have read and approved the final manuscript.

\section{Funding}

This study was supported by grants from the Nature Science Foundation of China (no. 81650014) and Beijing Natural Science Foundation (no. 7202034).

\section{Availability of data and materials}

All data generated or analyzed during this study are basically included in this published article (and its supplementary information files).

\section{Ethics approval and concent to participate}


Written informed consent was obtained from all participants according to the guidelines of the Declaration of Helsinki. All the collection of specimens were conducted following the Guidelines of Beijing You-an Hospital Capital Medical University.

\section{Consent for publication}

Not applicable.

\section{Competing interests}

The authors declare that they have no competing interests.

\section{References}

1. Steed E, Rodrigues NT, Balda MS, Matter K. Identification of MarvelD3 as a tight junction-associated transmembrane protein of the occludin family. BMC Cell Biol. 2009;10:95.

2. Cording J, Berg J, Kading N, Bellmann C, Tscheik C, Westphal JK, Milatz S, Gunzel D, Wolburg H, Piontek $\mathrm{J}$, et al. In tight junctions, claudins regulate the interactions between occludin, tricellulin and marvelD3, which, inversely, modulate claudin oligomerization. J Cell Sci. 2013;126(Pt 2):554-64.

3. Raleigh DR, Marchiando AM, Zhang Y, Shen L, Sasaki H, Wang Y, Long M, Turner JR. Tight junctionassociated MARVEL proteins marveld3, tricellulin, and occludin have distinct but overlapping functions. Mol Biol Cell. 2010;21(7):1200-13.

4. Balda MS, Matter K. Tight junctions and the regulation of gene expression. Biochim Biophys Acta. 2009;1788(4):761-7.

5. Martin TA, Mason MD, Jiang WG. Tight junctions in cancer metastasis. Front Biosci (Landmark Ed). 2011;16:898-936.

6. Kojima T, Takasawa A, Kyuno D, Ito T, Yamaguchi H, Hirata K, Tsujiwaki M, Murata M, Tanaka S, Sawada N. Downregulation of tight junction-associated MARVEL protein marvelD3 during epithelialmesenchymal transition in human pancreatic cancer cells. Exp Cell Res. 2011;317(16):2288-98.

7. Korompay A, Borka K, Lotz G, Somoracz A, Torzsok P, Erdelyi-Belle B, Kenessey I, Baranyai Z, Zsoldos $F$, Kupcsulik $P$, et al. Tricellulin expression in normal and neoplastic human pancreas. Histopathology. 2012;60(6B):E76-86.

8. Martin TA, Mansel RE, Jiang WG. Loss of occludin leads to the progression of human breast cancer. Int J Mol Med. 2010;26(5):723-34.

9. Jiang L, Yang YD, Fu L, Xu W, Liu D, Liang Q, Zhang X, Xu L, Guan XY, Wu B, et al. CLDN3 inhibits cancer aggressiveness via Wnt-EMT signaling and is a potential prognostic biomarker for hepatocellular carcinoma. Oncotarget. 2014;5(17):7663-76.

10. Steed E, Elbediwy A, Vacca B, Dupasquier S, Hemkemeyer SA, Suddason T, Costa AC, Beaudry JB, Zihni C, Gallagher E, et al. MarvelD3 couples tight junctions to the MEKK1-JNK pathway to regulate cell behavior and survival. J Cell Biol. 2014;204(5):821-38. 
11. Romani C, Cocco E, Bignotti E, Moratto D, Bugatti A, Todeschini P, Bandiera E, Tassi R, Zanotti L, Pecorelli $S$, et al. Evaluation of a novel human IgG1 anti-claudin3 antibody that specifically recognizes its aberrantly localized antigen in ovarian cancer cells and that is suitable for selective drug delivery. Oncotarget. 2015;6(33):34617-28.

12. Heerma van Voss MR, van Diest PJ, Smolders YH, Bart J, van der Wall E, van der Groep P. Distinct claudin expression characterizes BRCA1-related breast cancer. Histopathology. 2014;65(6):814-27.

13. Montgomery E, Mamelak AJ, Gibson M, Maitra A, Sheikh S, Amr SS, Yang S, Brock M, Forastiere A, Zhang $S$, et al. Overexpression of claudin proteins in esophageal adenocarcinoma and its precursor lesions. Appl Immunohistochem Mol Morphol. 2006;14(1):24-30.

14. Walther W, Petkov S, Kuvardina ON, Aumann J, Kobelt D, Fichtner I, Lemm M, Piontek J, Blasig IE, Stein U, et al. Novel Clostridium perfringens enterotoxin suicide gene therapy for selective treatment of claudin-3- and - 4-overexpressing tumors. Gene Ther. 2012;19(5):494-503.

15. Budhu A, Forgues M, Ye QH, Jia HL, He P, Zanetti KA, Kammula US, Chen Y, Qin LX, Tang ZY, et al. Prediction of venous metastases, recurrence, and prognosis in hepatocellular carcinoma based on a unique immune response signature of the liver microenvironment. Cancer Cell. 2006;10(2):99-111.

16. van Zijl F, Zulehner G, Petz M, Schneller D, Kornauth C, Hau M, Machat G, Grubinger M, Huber H, Mikulits W. Epithelial-mesenchymal transition in hepatocellular carcinoma. Future Oncol. 2009;5(8):1169-79.

17. Thiery JP. Epithelial-mesenchymal transitions in tumour progression. Nat Rev Cancer. 2002;2(6):442-54.

18. Yang G, Liang Y, Zheng T, Song R, Wang J, Shi H, Sun B, Xie C, Li Y, Han J, et al. FCN2 inhibits epithelial-mesenchymal transition-induced metastasis of hepatocellular carcinoma via TGFbeta/Smad signaling. Cancer Lett. 2016;378(2):80-6.

19. Xu MY, Chen R, Yu JX, Liu T, Qu Y, Lu LG. AZGP1 suppresses epithelial-to-mesenchymal transition and hepatic carcinogenesis by blocking TGFbeta1-ERK2 pathways. Cancer Lett. 2016;374(2):241-9.

20. Deng G, Zeng S, Ma J, Zhang Y, Qu Y, Han Y, Yin L, Cai C, Guo C, Shen H. The anti-tumor activities of Neferine on cell invasion and oxaliplatin sensitivity regulated by EMT via Snail signaling in hepatocellular carcinoma. Sci Rep. 2017;7:41616.

21. Cao F, Yin LX. PAK1 promotes proliferation, migration and invasion of hepatocellular carcinoma by facilitating EMT via directly up-regulating Snail. Genomics. 2020;112(1):694-702.

22. Che J, Yue D, Zhang B, Zhang H, Huo Y, Gao L, Zhen H, Yang Y, Cao B. Claudin-3 Inhibits Lung Squamous Cell Carcinoma Cell Epithelial-mesenchymal Transition and Invasion via Suppression of the Wnt/beta-catenin Signaling Pathway. Int J Med Sci. 2018;15(4):339-51.

23. Zhou W, Wang Q, Xu Y, Jiang J, Guo J, Yu H, Wei W. RMP promotes epithelial-mesenchymal transition through NF-kappaB/CSN2/Snail pathway in hepatocellular carcinoma. Oncotarget. 2017;8(25):40373-88.

24. Li Y, Wang LH, Zhang HT, Wang YT, Liu S, Zhou WL, Yuan XZ, Li TY, Wu CF, Yang JY. Disulfiram combined with copper inhibits metastasis and epithelial-mesenchymal transition in hepatocellular 
carcinoma through the NF-kappaB and TGF-beta pathways. J Cell Mol Med. 2018;22(1):439-51.

25. Pan JX, Chen G, Li JJ, Zhu QD, Li JJ, Chen ZJ, Yu ZP, Ye LY. Isocorydine suppresses doxorubicininduced epithelial-mesenchymal transition via inhibition of ERK signaling pathways in hepatocellular carcinoma. Am J Cancer Res. 2018;8(1):154-64.

26. Zhang G, Zhang G. Upregulation of FoxP4 in HCC promotes migration and invasion through regulation of EMT. Oncol Lett. 2019;17(4):3944-51.

27. Xue H, Tian GY. MiR-429 regulates the metastasis and EMT of HCC cells through targeting RAB23. Arch Biochem Biophys. 2018;637:48-55.

28. Thiery JP, Acloque H, Huang RY, Nieto MA. Epithelial-mesenchymal transitions in development and disease. Cell. 2009;139(5):871-90.

29. Luedde T, Schwabe RF. NF-kappaB in the liver-linking injury, fibrosis and hepatocellular carcinoma. Nat Rev Gastroenterol Hepatol. 2011;8(2):108-18.

30. Duhachek-Muggy S, Qi Y, Wise R, Alyahya L, Li H, Hodge J, Zolkiewska A. Metalloprotease-disintegrin ADAM12 actively promotes the stem cell-like phenotype in claudin-low breast cancer. Mol Cancer. 2017;16(1):32.

31. Li CF, Chen JY, Ho YH, Hsu WH, Wu LC, Lan HY, Hsu DS, Tai SK, Chang YC, Yang MH. Snail-induced claudin-11 prompts collective migration for tumour progression. Nat Cell Biol. 2019;21(2):251-62.

32. Bekes I, Friedl TW, Kohler T, Mobus V, Janni W, Wockel A, Wulff C. Does VEGF facilitate local tumor growth and spread into the abdominal cavity by suppressing endothelial cell adhesion, thus increasing vascular peritoneal permeability followed by ascites production in ovarian cancer? Mol Cancer. 2016;15:13.

33. Zeisel MB, Dhawan P, Baumert TF. Tight junction proteins in gastrointestinal and liver disease. Gut 2018.

34. Giannelli G, Koudelkova P, Dituri F, Mikulits W. Role of epithelial to mesenchymal transition in hepatocellular carcinoma. J Hepatol. 2016;65(4):798-808.

35. Zhang Y, Huang W. Transforming Growth Factor beta1 (TGF-beta1)-Stimulated Integrin-Linked Kinase (ILK) Regulates Migration and Epithelial-Mesenchymal Transition (EMT) of Human Lens Epithelial Cells via Nuclear Factor kappaB (NF-kappaB). Med Sci Monit. 2018;24:7424-30.

36. Lin L, Li R, Cai M, Huang J, Huang W, Guo Y, Yang L, Yang G, Lan T, Zhu K. Andrographolide Ameliorates Liver Fibrosis in Mice: Involvement of TLR4/NF-kappaB and TGF-beta1/Smad2 Signaling Pathways. Oxid Med Cell Longev. 2018;2018:7808656.

37. Yang Q, Xie B, Tang H, Meng W, Jia C, Zhang X, Zhang Y, Zhang J, Li H, Fu B. Minichromosome maintenance 3 promotes hepatocellular carcinoma radioresistance by activating the NF-kappaB pathway. J Exp Clin Cancer Res. 2019;38(1):263.

\section{Figures}


A

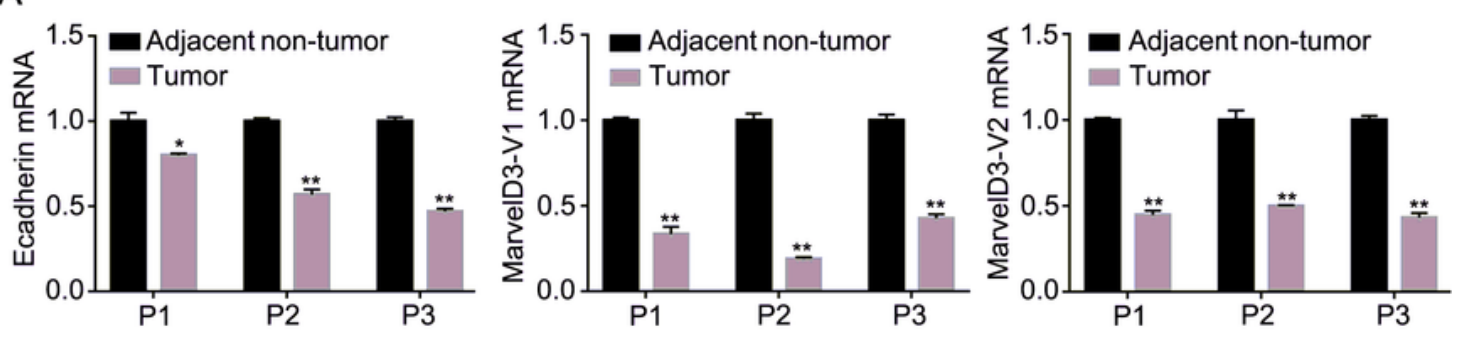

B

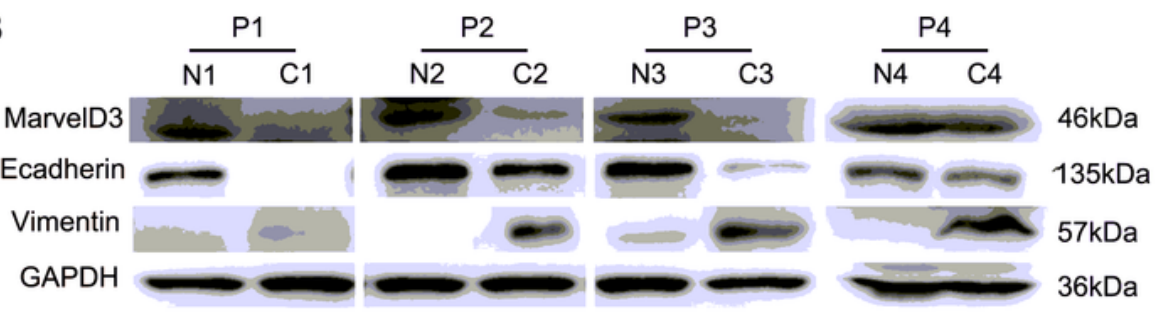

C

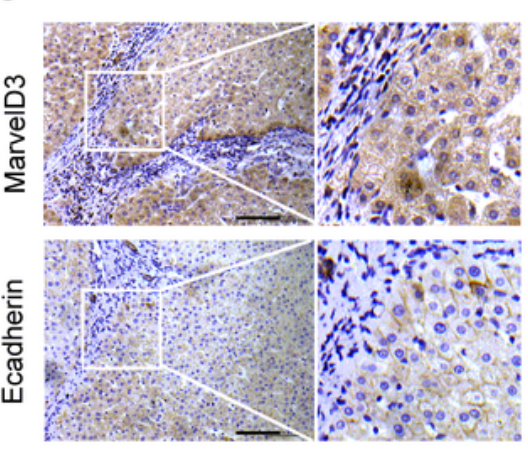

Normal

E

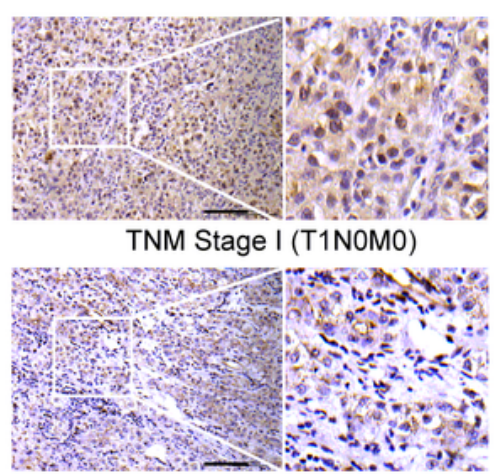

TNM Stage II (T2NOMO)

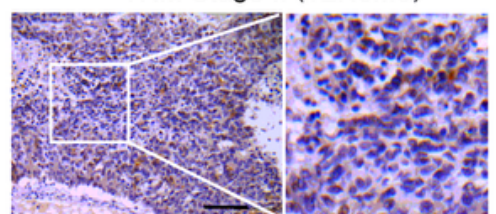

TNM Stage III (T3NOMO)
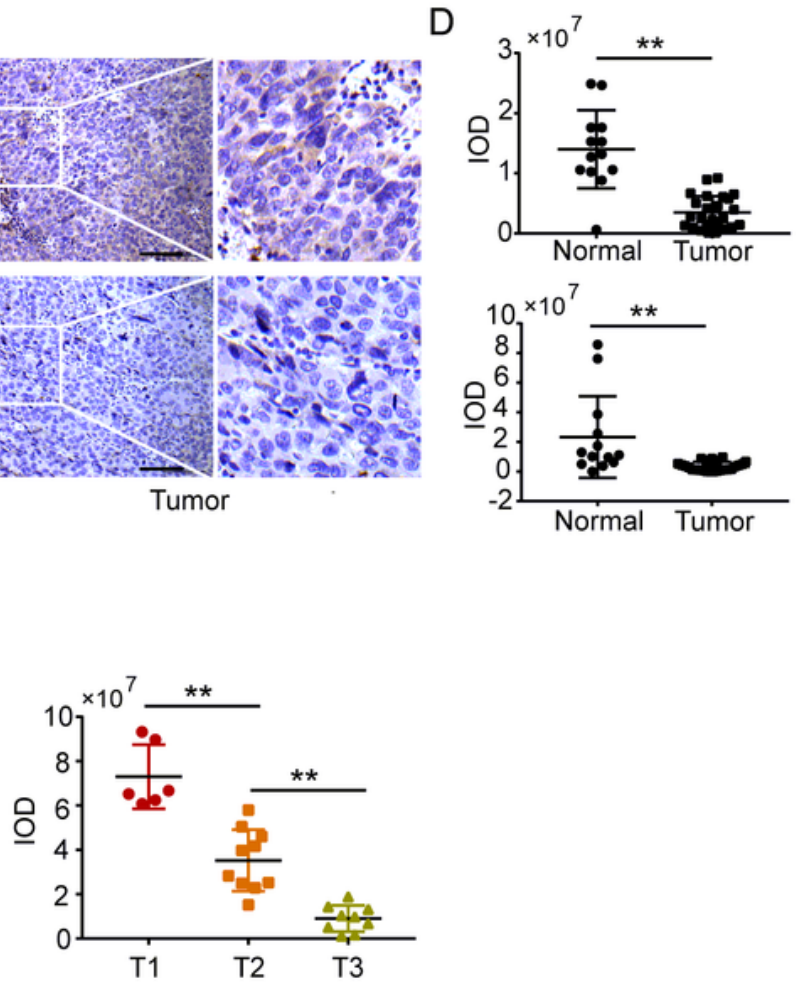

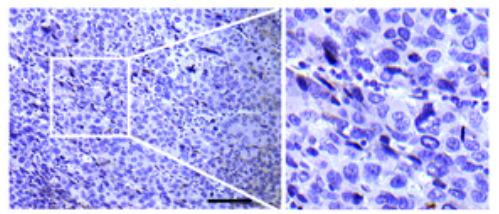

Tumor

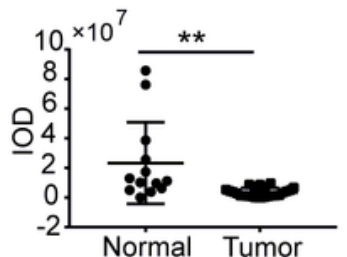

\section{Figure 1}

Association of reduced marvelD3 expression with altered EMT markers in HCC tissues compared with adjacent normal tissues (A) The expression of E-cadherin and MarvelD3 mRNA was determined by RTPCR in 3 pairs of HCC tissues; (B) The expression of E-cadherin, Vimentin and MarvelD3 protein were analyzed by western blotting in 4 pairs of HCC tissues; (N: normal liver tissues; C: HCC tissues); (C) Representative IHC images of E-cadherin and MarvelD3 staining in HCC tumor or normal liver tissues 
(scale bar, $100 \mu \mathrm{m}$; magnification, $200 \mathrm{X}$ and $400 \mathrm{X}$ ); (D) IHC scores of normal liver tissue ( $\mathrm{n}=13$ ) and liver tumor tissue ( $n=25)$ on E-cadherin and MarvelD3 staining; (E) MarvelD3 IHC staining and score in different TNM stage HCC tumor were analyzed.

A
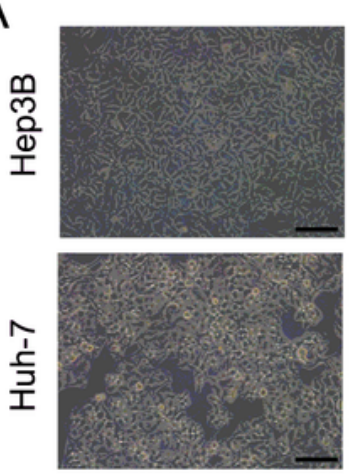

control

C
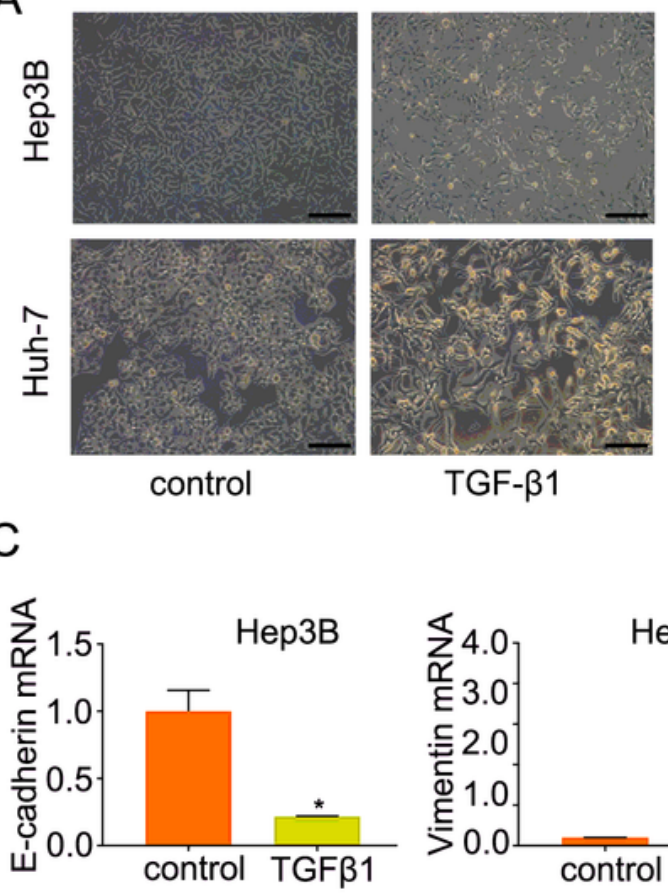

TGF- $\beta 1$

B

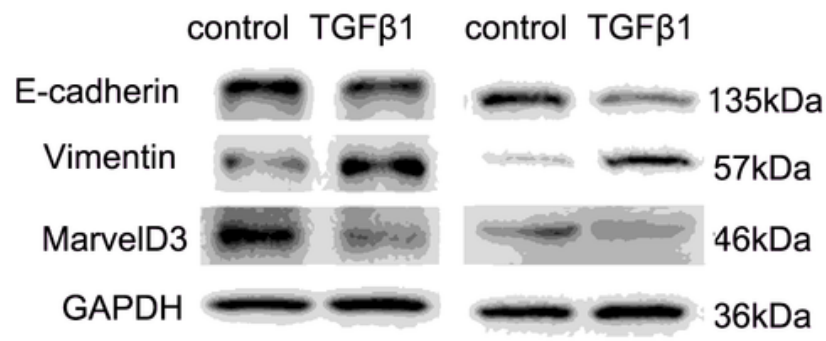

Hep3B

Huh-7
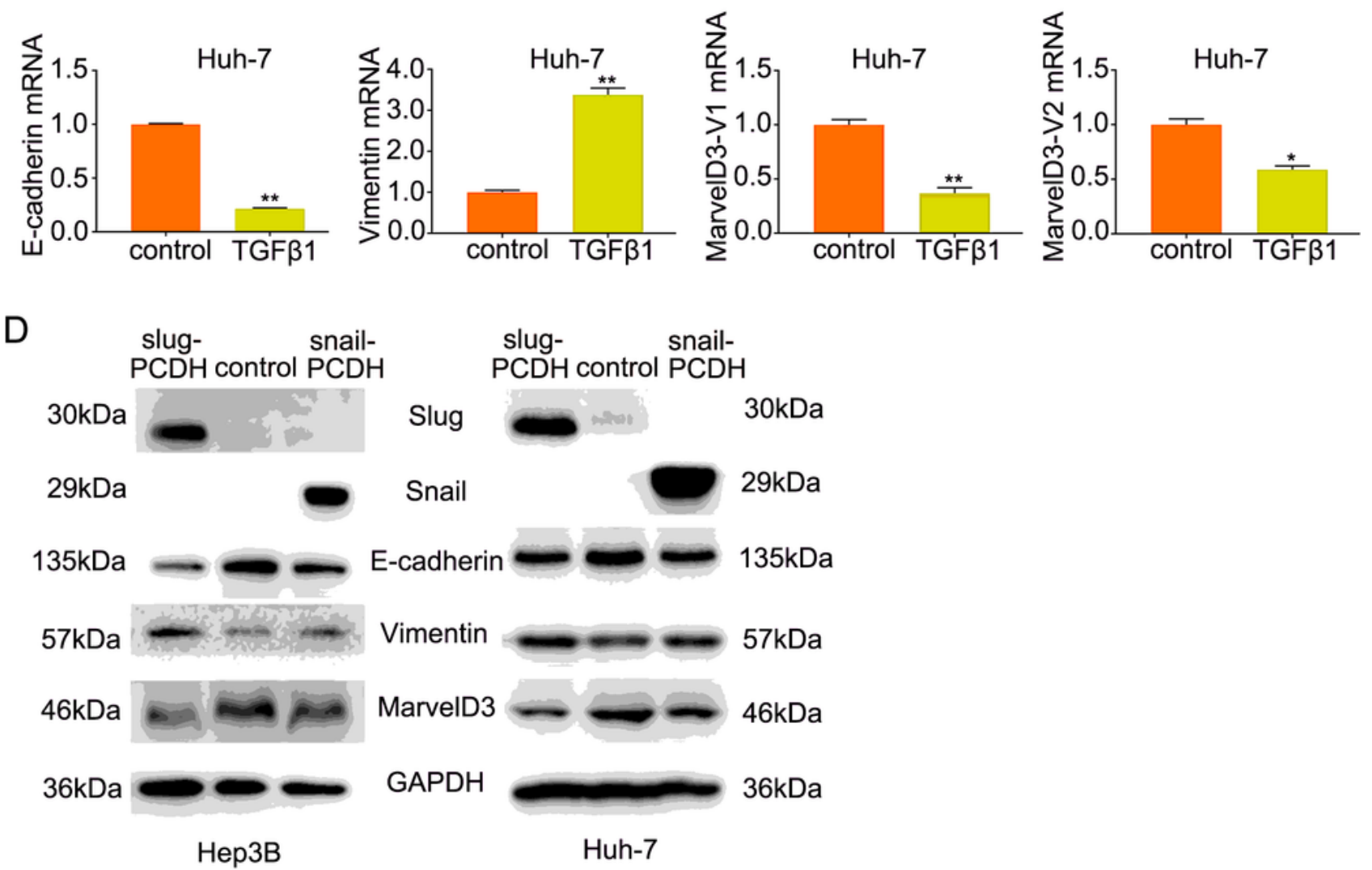

Нер3B
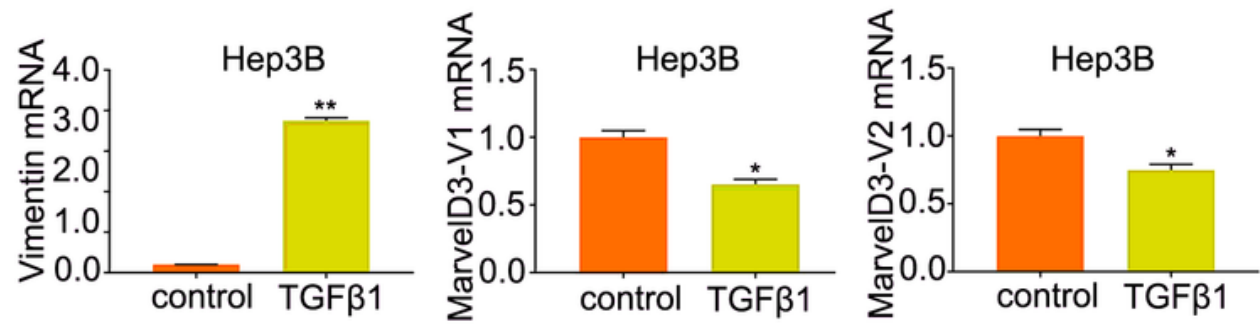

Figure 2

Downregulation of marvelD3 during TGF- $\beta 1$ and snail-induced EMT in HCC cells (A) HCC cells stimulated with TGF- $\beta 1$ exhibited morphological changes into spindle-shaped cells with loose cell connections. 
Observations were made using a light microscope $(\times 100)$. (B) and (C) The expression of E-cadherin, Vimentin and MarvelD3 was tested in HCC cells treated with TGF- $\beta 1$ by western blot (B) and RT-PCR (C). (D) The expression of E-cadherin, Vimentin and MarvelD3 was evaluated in snail- and slug-transfected HCC cells by western blot. $\left({ }^{*} P<0.05, * * P<0.01\right)$

A

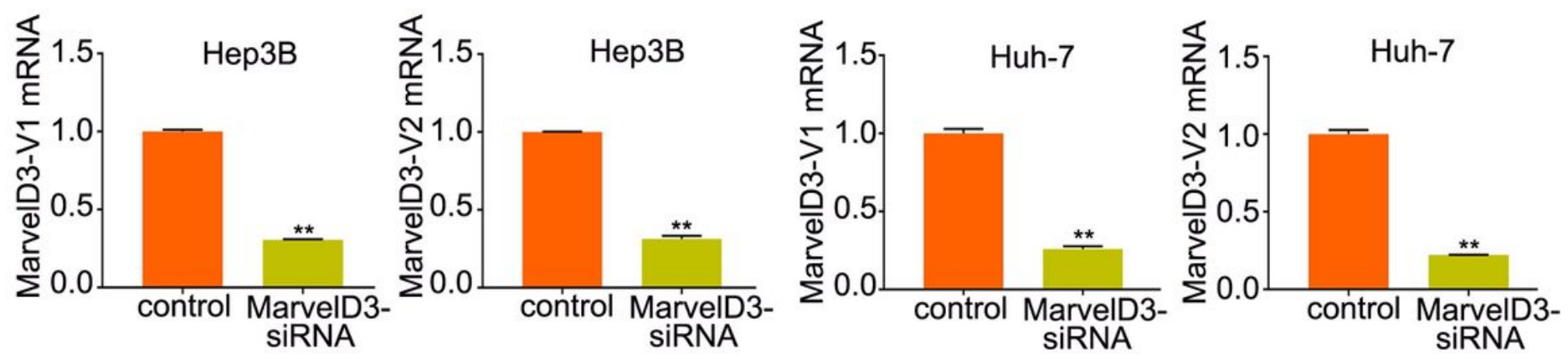

B

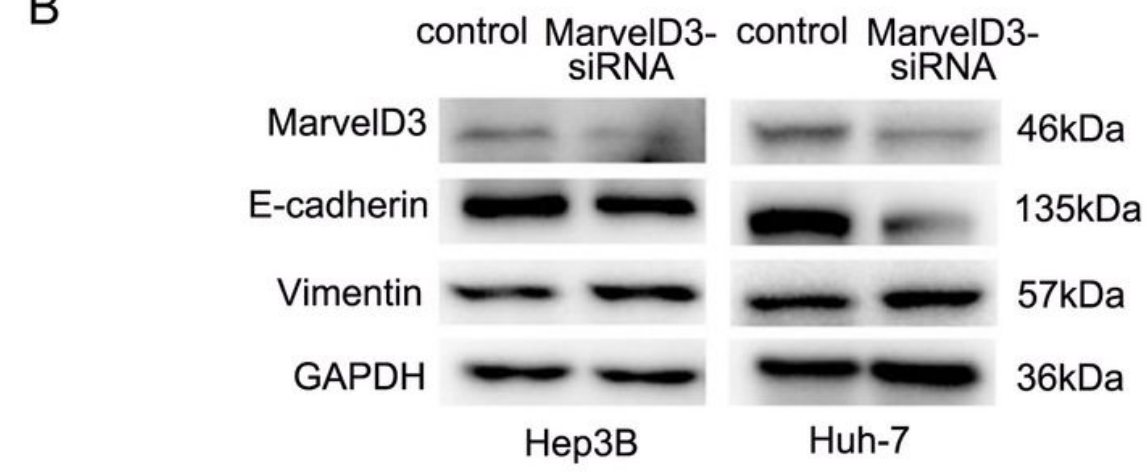

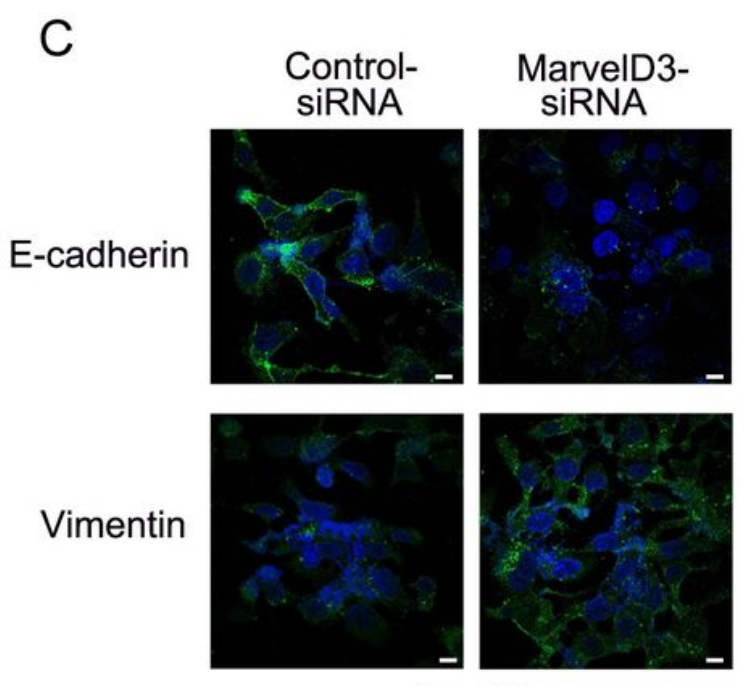

Hep3B

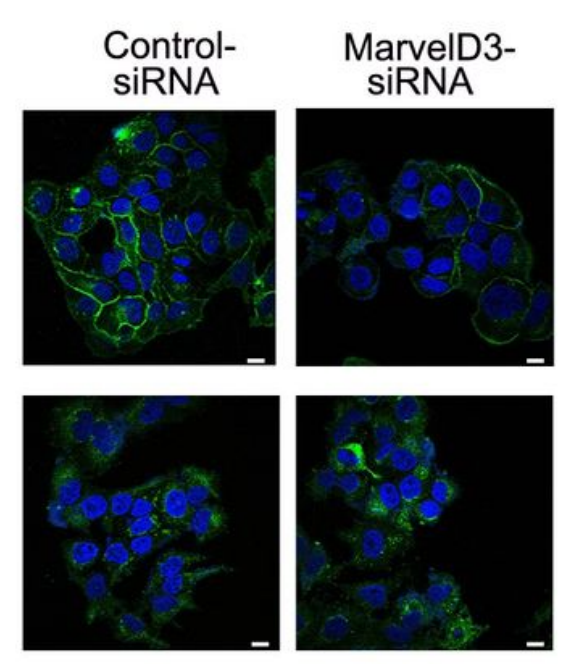

Huh-7

\section{Figure 3}

Effects of marvelD3 knockdown on regulation of EMT gene expression in vitro (A) RT-PCR was performed to examine the marvelD3 mRNA level. (B) MarvelD3, E-cadherin and Vimentin expression were assayed by western blot. (C) Immunofluorescence images show that marvelD3-knockdown downregulated the expression of E-cadherin and upregulated the expression of Vimentin (scale bar, $50 \mu \mathrm{m}$; magnification, $400 \mathrm{X}) .\left({ }^{*} \mathrm{P}<0.05,{ }^{*} \mathrm{P}<0.01\right)$ 


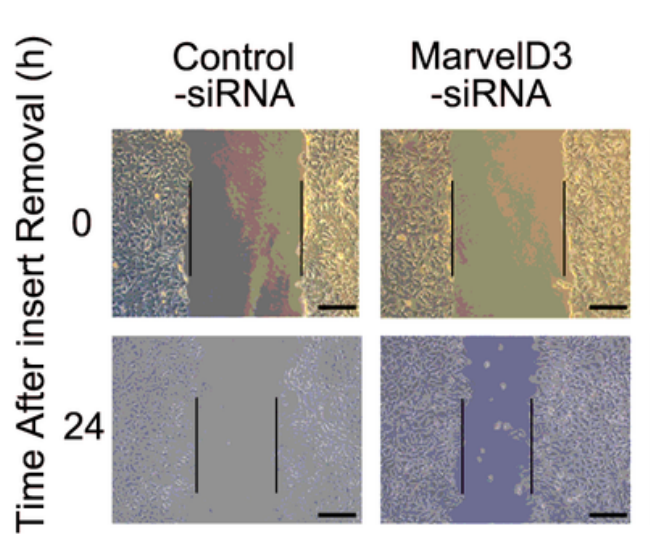

Нер3B

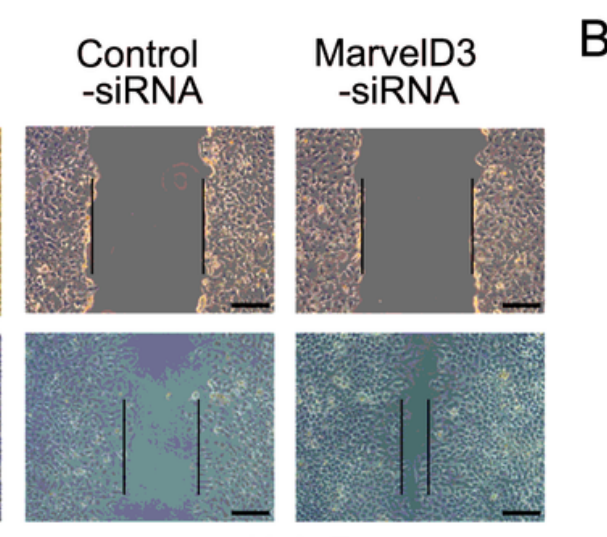

Huh-7

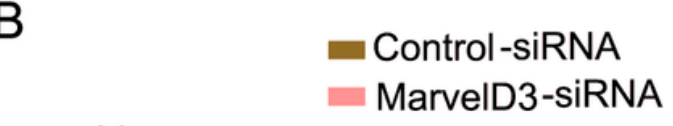

C

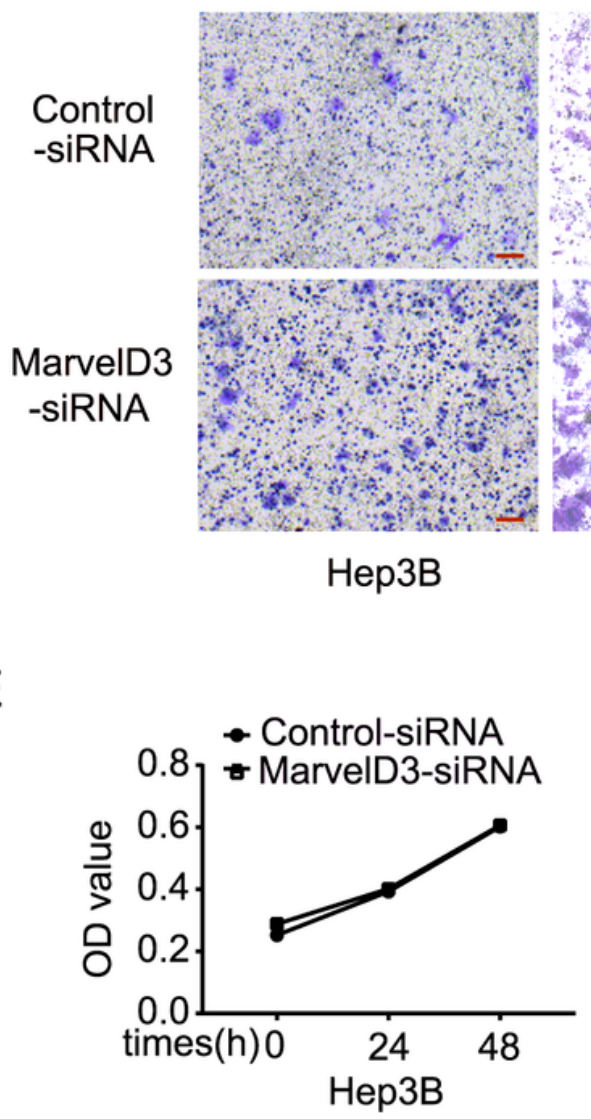

$\mathrm{D}$



\section{Figure 4}

Effects of marvelD3 knockdown on HCC cell migration in vitro (A) Wound healing assay was used to detected the wound healing capacity in HCC cells treated with or without marvelD3 knockdown (100 X). (B) The bar chart showed healing ratio. (C) and (D) Representative images from the transwell migration assay in marvelD3-knockdown HCC cells. Magnification, 100x. Scale, $100 \mu \mathrm{m}$. Data represent the mean $\pm S D$. ${ }^{*} P<0.05,{ }^{*} P<0.01$. (E) The proliferation capacities of marvelD3-knockdown HCC cells were detected by MTS.

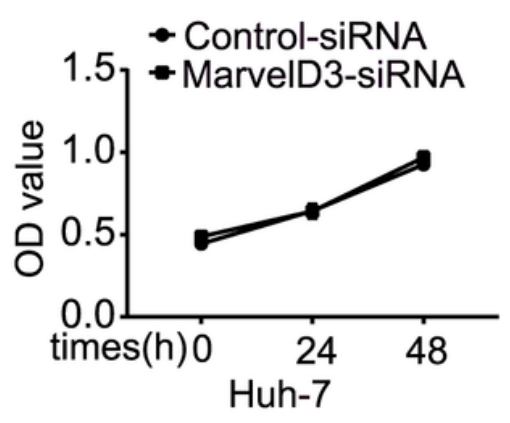






Figure 5

Effects of marvelD3 on inactivation of the NF-kB signaling pathway (A) NF-kB pathway proteins including Ikka, IkBa, NF-kB and MMP9 were measured by western blot in the indicated HCC cells where MarvelD3 was knocked down. (B) NF-kB pathway inihibitor BAY 11-7082 was applied to marvelD3-knockdown HCC cells. Western blot was used to verify the expression of NF-kB. (C) Representative images from the transwell migration assay demonstrated the migration capacity in marvelD3-knockdown HCC cells treated with NF-kB pathway inihibitor BAY 11-7082, with bar charts showing migration numbers (right panel). Magnification, 100×. Scale, $100 \mu \mathrm{m} .\left({ }^{\star} P<0.05,{ }^{\star *} \mathrm{P}<0.01\right)$.

\section{Supplementary Files}

This is a list of supplementary files associated with this preprint. Click to download. 
- SupplementaryMaterials.docx

Page 19/19 